\title{
Neue Synthesen von 2.4-disubstituierten s-Triazinen
}

Von Prof, Dr. H. Bredereck, Dr. F. Effenberger und Dipl.-Chem. Alfred Hofmann

Institut für Organische Chemic und Organisch-Chemische Technologie der T.H. Stuttgart

Bei Synthesen von s-Triazin und $\mathrm{N}$-substituicrten s-Triazinen [1] haben wir die Darstellung des 2.4-Diamino-s-triazins u. a. aus Guanidincarbonat und Formamid beschrieben. Wir konnten jetzt durch Umsetzung von Harnstoff, Guanidin, substituierten Guanidinen, Benzamidin und S-Methyl-iso-thioharnstoff mit Formylierungsmitteln (Trisformaminomethan, Orthoameisensä ureester, Dimethylformamid - diäthylacetal) in guten Ausbeuten zahlreiche 2.4-disubstituierte Triazine crhalten.

Erhitzen von Harnstoff mit Trisformaminomethan oder Orthoameisensäureester führte zu dem bisher nur sehr schwer zugänglichen [2] 2.4-Dihydroxy-s-triazin.

Guanidin ergab mit Dimethylformamid-diäthylacetal 2.4 Diamino-s-triazin und entspr. Butylguanidin das 2.4-Bisbutylamino-s-triazin, Phenylguanidin das 2.4-Dianilino-striazin und p-Tolylguanidin das 2.4-Di-p-toluidino-s-triazin. Die Umsetzung von Benzamidin mit Ameisensäureäthylester oder Dimethylformamid-diäthylacetal führte zum 2.4-Diphenyl-s-triazin. Schließlich lieferte S-Methyl-iso-thioharnstoffhydrochlorid durch Erhitzen mit Dimethylformamiddiäthylacetal das bereits fruher von uns beschriebene 2-Methylmercapto-4-amino-s-triazin.

[1] H. Bredereck, O. Smerz u. R. Gompper, Chem. Ber. 94,1883 (196I).

[2] I. Flament, R. Promel u. R. H. Martin, Helv, chim. Acta 42, 487 (1959); H. Bittz u. E. Gieseler, Ber. dtsch. chem. Ges. 46, 3410 (1913). 